\title{
Vascular Flora of the Keystone Wildlife Management Area, Creek, Pawnee, and Osage Counties, Oklahoma
}

\author{
Bruce W. Hoagland \\ Oklahoma Biological Survey \\ and Department of Geography \\ University of Oklahoma \\ Norman, OK 73019
}

\author{
Amy K. Buthod \\ Oklahoma Biological Survey \\ University of Oklahoma \\ Norman, OK 73019
}

This paper reports the results of an inventory of the vascular plants at the Keystone Wildlife Management Area in northeastern Oklahoma. A total of $\mathbf{3 8 0}$ taxa of vascular plants in $\mathbf{2 5 4}$ genera and $\mathbf{7 9}$ families were collected. The most species were collected from the families Poaceae (58), Asteraceae (57), and Fabaceae (30). There were 160 annual and 220 perennial species. Fifty-six species of woody plants were present. A total of 59 exotic species were collected representing $15 \%$ of the flora. No species tracked by the Oklahoma Natural Heritage Inventory for rarity were found.

\section{INTRODUCTION}

Floristic inventories can be undertaken to address a number of research or management objectives. For example, florisitic inventories are often necessary when analyzing species distributions. Often there are gaps in the known geographic distribution of species and groups of species, so an inventory maybe required in order to complete distribution maps. Inventories are also crucial in plant conservation in order to locate populations of rare or threatened species. Finally, inventories aid land managers in both the protection of sensitive species and the location of nuisance species. Floristic inventories can be conducted at either the regional or local scale. The objective of this study was to provide a floristic inventory to aid Oklahoma
Department of Wildlife Conservation personnel in management of the Keystone Wildlife Management Area (KWMA).

\section{STUDY AREA}

The KWMA is located along the upper reaches of Keystone Lake, which was flooded in 1964 (Oklahoma Water Resources Board 1990). Keystone Dam is situated just below the confluence of the Cimarron and Arkansas Rivers, both of which flow through KWMA. The KWMA was established in 1973 by the Oklahoma Department of Wildlife Conservation and is comprised of over 16,000 acres in Creek, Osage, and Pawnee Counties (Pennington 2003).

The KWMA is located within the Subtropical Humid (Cf) climate zone

Hoagland, B.W. and Buthod, A.K. https://doi.org/10.22488/okstate.17.100020 
(Trewartha 1968). Summers are warm (mean July temperature $=27.3^{\circ} \mathrm{C}$ ) and humid, and winters are relatively short and mild (mean January temperature $=2.6^{\circ} \mathrm{C}$ ). Mean annual precipitation is $98 \mathrm{~cm}$ with periodic severe droughts (Oklahoma Climatological Survey 2003).

Physiographically, the study area is located in the Osage Plains section of the Central Lowlands province (Hunt 1974) and within the Eastern Sandstone Cuesta plains of Oklahoma (Curtis and Ham 1979). The surface geology consists primarily of Pennsylvanian sandstone and shale (Branson and Johnson 1979). Outcroppings of silty sandstones occur in the eastern portion of KWMA with sandy, silty shales and limestone outcrops in the west (Grieg 1959, Oakes and Jordan 1959). The soils at KWMA are sandy loams and silt loams. Bottomland soils include Yahola very fine sandy loam and Reinach very sandy loam. Uplands soils are steep to gently rolling and consist primarily of the Eufaula loamy fine sand (Oakes 1959). The predominant potential vegetation types are post oakblackjack and bottomland forests (Duck and Fletcher 1943). However, much of the bottomland forest area has been converted to agriculture and is now actively cultivated or in old-fields. Upland forests have been heavily modified as well.

\section{METHODS}

Collections were also made randomly throughout the KWMA from March through October 2002. Sixteen collection sites were established to represent the greatest variety of habitats for intensive floristic sampling. Sites were selected following a review of U. S. Geological Survey 1:24,000 topographic maps and field reconnaissance. Vouchers for exotic species were made from naturalized populations only, thus excluding cultivated and ornamental plants. Specimens were processed and a voucher set was deposited at the Robert Bebb Herbarium of the University of Oklahoma (OKL) following standard curatorial procedures (Bridson 1992). Manuals used for specimen identification included Keys to the Flora of Oklahoma (Waterfall 1969), Flora of the Great Plains (Barkley 1986), Shinners \& Mahler's Illustrated Flora of North Central Texas (Diggs et al 1999), and Steyermark's Flora of Missouri (Yatsievych 1999). Origin and nativity for species was determined by reference to Taylor and Taylor (1991) and the United States Department of Agriculture-Natural Resources Conservation Service (USDANRCS 2003). Nomenclature follows USDA-NRCS (2003).

\section{RESULTS AND DISCUSSION}

A total of 380 taxa of vascular plants in 254 genera and 79 families were collected. Among the angiosperms, 294 were dicots and 83 were monocots. In addition, there were two ferns, and one gymnosperm. The Poaceae (58), Asteraceae (57), and Fabaceae (30) had the greatest numbers of species. The genera Polygonum (8), Quercus (7), Carex (6), Desmodium (6), and Eragrostis (6) contained the greatest numbers of species. There were 160 annual and 220 perennial species. Fifty-six species were woody plants, 30 of which were trees, 11 shrubs, and 15 vines.

Fifteen percent of the flora was exotic. A total of 59 exotic species were collected in 20 families. The greatest numbers of exotic species were in the 
Poaceae (12) and Fabaceae (8). These families also had the greatest number of exotic species at the Chickasaw National Recreation Area (Hoagland and Johnson 2001). In that study, $12 \%$ of the flora was composed of exotic species. Six of the eight species reported in the Caryophyllaceae were exotic, the highest ratio for any family. The greatest number of introduced species was in the genus Bromus (4). No species tracked by the Oklahoma Natural Heritage Inventory for rarity were encountered.

\section{ACKNOWLEDGMENTS}

I express my sincere gratitude to my major advisor, Dr. Ronald J. Tyrl for his invaluable guidance, encouragement, honesty, and friendship. I thank my other committee members, Dr. Paul Buck and Dr. Rahmona Thompson for their assistance with my project. Thanks also goes to The Nature Conservancy and Nora Jones. Finally, I thank the personnel of the Botany Department, the NABS lab, and Lou Edith Hara for their assistance.

\section{LITERATURE CITED}

Barkley, T. M. (ed.) 1986. Flora of the Great Plains. Lawrence, KS: University Press of Kansas.

Branson, C.C., and K.S. Johnson. 1979. Generalized geologic map of Oklahoma. In: Johnson K.S., et al., editors. Geology and earth resources of Oklahoma. Norman, OK: Oklahoma Geological Survey.

Bridson, D. M. 1992. The herbarium handbook. Royal Botanical Gardens at Kew, England.

Curtis, N. M. and W. E. Ham. 1979. Geomorphic provinces of Oklahoma.
In: Johnson, K.S., et al., eds. Geology and earth resources of Oklahoma. Norman, OK: Oklahoma Geological Survey.

Diggs, George M., Barney L. Lipscomb, and Robert J. O'Kennon. 1999.

Shinners and Mahler's illustrated flora of North Central Texas. Fort Worth, TX: Botanical Research Institute of Texas and Austin College.

Duck, L.G., and J.B. Fletcher. 1943. Game type map of Oklahoma. Oklahoma City, OK: Oklahoma Department of Wildlife Conservation.

Grieg, Paul B. 1959. Geology of Pawnee County, Oklahoma. Norman, OK: Oklahoma Geological Survey.

Hoagland, B.W. and F.L. Johnson. 2001. Vascular flora of the Chickasaw National Recreation Area, Murray County, Oklahoma. Castanea 66: 383-400.

Hunt, C.B. 1974. Natural regions of the United States and Canada. San Francisco, CA: W. H. Freeman.

Oakes, H. 1959. Soil survey of Creek County, Oklahoma. United States Department of Agriculture.

Oakes, M.C. and L. Jordan. 1959. Geology of Creek County. Norman, OK: Oklahoma Geological Survey.

Oklahoma Climatological Survey. 2003. Oklahoma climatological data. (http://www.ocs.ou.edu/).

Oklahoma Water Resources Board. 1990. Oklahoma water atlas. Oklahoma Water Resources Publication 135. Oklahoma City, OK

Pennington, Jeff. 2003. Keystone wildlife management area. www.wildlifedepartment.com/keysto 
ne.htm, Oklahoma Department of Wildlife Conservation.

Taylor, R. John, and Constance E. S. Taylor. 1991. An annotated list of the ferns, fern allies, gymnosperms, and flowering plants of Oklahoma. [Published by the authors at Southeastern Oklahoma State University.

Trewartha, G. T. 1968. An introduction to climate. $4^{\text {th }}$ ed. New York: McGrawHill.
USDA-NRCS 2003. The PLANTS database. (http://plants.usda.gov/plants). National Plant Data Center, Baton Rouge, LA

Waterfall, U. T. 1969. Keys to the flora of Oklahoma. $4^{\text {th }}$ ed. Stillwater, OK: [Published by the author].

Yatsievych, George. 1999. Steyermark's flora of Missouri, Vol. 1, rev. ed. St. Louis, MO: Missouri Department of Conservation in cooperation with the Missouri Botanical Garden Press. 


\section{Keystone Wildlife Management Area Annotated Species List}

Growth habit is designated as F; forb, G; graminoid, H; herb, S; shrub, T; tree, and V; woody vine. Life history is designated as A; annual/biennial or P; perennial. Origin is noted as $\mathrm{N}$; native or I; introduced.

\section{Pteridophyta (Ferns and allies)}

\section{Aspleniaceae}

Asplenium platyneuron (L.) B.S.P. ebony spleenwort; F or H, P, N

Dryopteridaceae

Woodsida obtusa (Spreng.) Torr. bluntlobe cliff fern; F or H, P, N

\section{Coniferophyta (Gymnosperms)}

\section{Cupressaceae}

Juniperus virginiana L. eastern redcedar; T, P, N

\section{Magnoliophyta (Angiosperms)}

\section{Monocotyledonae}

\section{Araceae}

Arisaema dracontium (L.) Schott green dragon; $\mathrm{F}$ or $\mathrm{H}, \mathrm{P}, \mathrm{N}$

\section{Alistmataceae}

Sagittaria montevidensis Cham. \& Schlect. giant arrowhead; F or H, P, I

\section{Commelinaceae}

Tradescantia ohiensis Raf. bluejacket; F or H, P, N

\section{Cyperaceae}

Carex albicans Willd. Ex Spreng. whitetinge sedge; G, P, N

Carex amphibola Steud. eastern narrowleaf sedge; G, P, N

Carex brevior (Dewey) Mackenzie shortbeak sedge; G, P, N
Carex bushii Mackenzie

$$
\text { Bush's sedge; G, P, N }
$$

Carex caroliniana Schwein.

Carolina sedge; G, P, N

Carex leavenworthii Dewey

Leavenworth's sedge; G, P, N

Cyperus croceus Vahl

Baldwin's flatsedge; G, P, N

Cyperus echinatus (L.) Wood

Globe flatsedge; G, P, N

Cyperus erythrorhizos Muhl.

redroot flatsedge; G, P, N

Cyperus lupulinus (Spreng.) Marcks

Great Plains flatsedge; G, P, N

Cyperus setigerus Torr. \& Hook.

lean flatsedge; G, P, N

Eleocharis engelmannii Steud.

Engelmann's spikerush; G, P, N

Fimbristylis puberula (Michx.) Vahl hairy fimbry; G, P, N

Iridaceae

Sisyrinchium angustifolium P. Mill. narrowleaf blue-eyed grass; F or $\mathrm{H}, \mathrm{P}, \mathrm{N}$

\section{Juncaceae}

Juncus brachyphyllus Wieg. tuftedstem rush; G, P, N

Juncus dudleyi Wieg.

Dudley's rush; G, P, N

Juncus marginatus Rostk.

\section{Liliaceae} grassleaf rush; G, P, N

Allium perdulce S.V. Fraser plains onion; F or $\mathrm{H}, \mathrm{P}, \mathrm{N}$ Erythronium sp. L. 
troutlily; F or $\mathrm{H}, \mathrm{P}, \mathrm{N}$

Nothoscordum bivalve (L.) Britt. crowpoison; F or $\mathrm{H}, \mathrm{P}, \mathrm{N}$

\section{Poaceae}

Agrostis hyemalis (Walt.) B.S.P. winter bentgrass; G, P, N

Alopecurus carolinianus Walt. Carolina foxtail; G, A, N

Andropogon gerardii Vitman big bluestem; G, P, N

Andropogon ternarius Michx. splitbeard bluestem; G, P, N

Bouteloua rigidiseta (Steud.) A.S. Hitchc. Texas grama; G, P, N

Bromus catharticus Vahl rescuegrass; G, A, I

Bromus hordeaceus L. soft brome; G, P, I

Bromus japonicus Thunb. ex Murr. Japanese brome; G, A, I

Bromus tectorum L. cheatgrass; G, A, I

Buchloe dactyloides (Nutt.) Engelm. buffalograss; G, P, N

Cenchrus spinifex Cav. coastal sandbur; G, P, N

Chasmanthium latifolium (Michx.) Yates Indian woodoats; G, P, N

Chloris verticillata Nutt. tumble windmill grass; G, P, N

Coelorachis cylindrica (Michx.) Nash cylinder jointtail grass; G, P, N

Cynodon dactylon (L.) Pers. Bermudagrass; G, P, I

Dichanthelium aciculare (Desv. ex Poir.) Gould \& C.A. Clark needleleaf rosette grass; $\mathrm{G}, \mathrm{P}, \mathrm{N}$

Dichanthelium acuminatum (Sw.) Gould \& C.A. Clark var. fasciculatum (Torr.) Freckmann western panicgrass; $\mathrm{G}, \mathrm{P}, \mathrm{N}$

Dichanthelium malacophyllum Nash (Gould) softleaf rosette grass; $\mathrm{G}, \mathrm{P}, \mathrm{N}$

Dichanthelium oligosanthes (J. A. Schultes)

Gould var. oligosanthes

Heller's rosette grass; G, P, N

Dichanthelium scoparium Lam. (Gould)

velvet panicum; G, P, N

Digitaria sanguinalis (L.) Scop. hairy crabgrass; G, A, N

Echinochloa crus-galli (L.) Beauv. barnyardgrass; G, A, I

Eleusine indica (L.) Gaertn. Indian goosegrass; G, A, I

Elymus virginicus L.

Virginia wildrye; G, P, N

Eragrostis barrelieri Daveau Mediterranean lovegrass; G, A, I

Eragrostis hirsuta (Michx.) Nees bigtop lovegrass; G, P, N

Eragrostis pilosa (L.) Beauv. Indian lovegrass; G, A, N

Eragrostis secundiflora J. Pesl red lovegrass; G, P, N

Eragrostis spectabilis (Pursh) Steud. purple lovegrass; G, P, N

Eragrostis trichodes (Nutt.) Wood sand lovegrass; G, P, N

Eriochloa contracta A.S. Hitchc. prairie cupgrass; G, A, N

Gymnopogon ambiguous (Michx.) B.S.P. bearded skeletongrass; G, P, N

Hordeum pusillum Nutt. little barley; G, A, N

Leersia virginica Willd. whitegrass; G, P, N

Leptochloa fusca (L.) Kunth Malabar sprangletop; G, P, N

Leptochloa panacea (Retz.) Ohwi ssp. mucronata (Michx.) Nowack mucronate sprangletop; G, A, N

Lolium arundinaceum (Schreb.) S.J.

Darbyshire tall fescue; G, P, I

Lolium perenne L.

Hoagland, B.W. and Buthod, A.K. 
Oklahoma Native Plant Record

Volume 3, Number 1, December 2003

perennial ryegrass; $\mathrm{G}, \mathrm{P}, \mathrm{I}$

Muhlenbergia frondosa (Poir.) Fern. wirestem muhly; G, P, N

Muhlenbergia sobolifera (Muhl. ex Willd.) Trin. rock muhly; $\mathrm{G}, \mathrm{P}, \mathrm{N}$

Neeragrostis reptans (Michx.) Nicora creeping lovegrass; G, A, N

Panicum anceps Michx. beaked panicgrass; G, P, N

Panicum philadelphicum Bernh. ex Trin. Philadelphia panicgrass; G, A, N

Panicum virgatum $\mathrm{L}$. switchgrass; G, P, N

Paspalum pubiflorum Rupr. ex Fourn. hairyseed paspalum; G, P, N

Paspalum setaceum Michx. thin paspalum; G, P, N

Poa annua L. annual bluegrass; G, A, I

Poa arachnifera Torr. Texas bluegrass; G, P, N

Poa pratensis L. Kentucky bluegrass; G, P, I

Schedonnardus paniculatus (Nutt.) Trel. Tumblegrass; G, P, N

Schizachyrium scoparium (Michx.) Nash little bluestem; G, P, N

Setaria pumila (Poir.) Roemer \& J.A. Schultes yellow bristlegrass; G, A, I

Sorghastrum nutans (L.) Nash Indiangrass; G, P, N

Sorghum halepense (L.) Pers. Johnsongrass; G, P, I

Sphenopholis obtusata (Michx.) Scribn. prairie wedgescale; G, P, N

Tridens flavus (L.) A.S. Hitchc. purpletop tridens; G, P, N

Tripsacum dactyloides (L.) L. eastern gamagrass; G, P, N

Vulpia sciurea (Nutt.) Henr. squirreltail fescue; $\mathrm{G}, \mathrm{A}, \mathrm{N}$

\section{Smilacaceae}

Smilax bona-nox L. saw greenbriar; S, SS, V, P, N

Smilax rotundifolia $\mathrm{L}$. roundleaf greenbriar; S, SS, V, P, N

\section{Dicotyledonae}

\section{Acanthaceae}

Dicliptera brachiata (Pursh) Spreng. branched foldwing; F or $\mathrm{H}, \mathrm{A}, \mathrm{N}$

Ruellia humilis Nutt. fringeleaf wild petunia; F or $\mathrm{H}, \mathrm{P}, \mathrm{N}$

Ruellia strepens L. limestone wild petunia; F or $\mathrm{H}, \mathrm{P}, \mathrm{N}$

\section{Aceraceae}

Acer negundo L.

boxelder; T, P, N

Acer saccharinum L. silvermaple; T, P, N

\section{Amaranthaceae}

Amaranthus rudis Sauer tall amaranth; F or $\mathrm{H}, \mathrm{A}, \mathrm{N}$

Froelichia floridana (Nutt.) Moq. plains snakecotton; F or $\mathrm{H}, \mathrm{A}, \mathrm{N}$

Froelichia gracilis (Hook.) Moq. slender snakecotton; F or H, A, N

Iresine rhizomatosa Standl. Juda's bush; F or H, P, N

Anacardiaceae

Rhus aromatica Ait.

fragrant sumac; S, P, N

Rhus copallinum L.

flameleaf sumac; T, S, P, N

Toxicodendron radicans (L.) Kuntze eastern poison ivy; S, SS, V, P, N

\section{Apiaceae}

Ammoselinum popei Torr. \& Gray plains sandparsley; F or $\mathrm{H}, \mathrm{A}, \mathrm{N}$ Chaerophyllum tainturieri Hook. 
hairyfruit chervil; F or $\mathrm{H}, \mathrm{A}, \mathrm{N}$

Cicuta maculata L.

spotted water hemlock; F or H, P, N

Daucus pusillus Michx.

American wild carrot; F or H, A, N

Eryngium leavenworthii Torr. \& Gray

Leavenworth's eryngo; F or H, A, N

Polytaenia nuttallii DC.

Nuttall's prairie parsley; F or H, P, N

Ptilimnium nuttallii (DC.) Britt.

laceflower; F or H, A, N

Sanicula canadensis L.

Canadian blacksnakeroot;

F or $\mathrm{H}, \mathrm{P}, \mathrm{N}$

Spermolepis divaricata (Walt). Raf. ex Ser.

roughfruit scaleseed; F or $\mathrm{H}, \mathrm{A}, \mathrm{N}$

Trepocarpus aethusae Nutt. ex DC. whitenymph; $\mathrm{F}$ or $\mathrm{H}, \mathrm{A}, \mathrm{N}$

Torilis arvensis (Huds.) Link spreading hedgeparsley; F or, H, A, I

Apocynaceae

Apocynum cannabinum $\mathrm{L}$. Indianhemp; F or $\mathrm{H}, \mathrm{P}, \mathrm{N}$

Aristolochiaceae

Aristolochia tomentosa Sims

wooly dutchman's pipe; V, P, N

Asclepiadaceae

Asclepias amplexicaulis $\mathrm{Sm}$.

clasping milkweed; F or $\mathrm{H}, \mathrm{P}, \mathrm{N}$

Asclepias tuberosa L.

butterfly milkweed; F or H, P, N

Asclepias viridis Walt.

green antelope horn; F or $\mathrm{H}, \mathrm{P}, \mathrm{N}$

Asteraceae

Achillea millefolium $\mathrm{L}$.

common yarrow; F or $\mathrm{H}, \mathrm{P}, \mathrm{N}$

Ambrosia artemisiifolia $\mathrm{L}$. annual ragweed; F or $\mathrm{H}, \mathrm{A}, \mathrm{N}$

Ambrosia trifida L.

great ragweed; $\mathrm{F}$ or $\mathrm{H}, \mathrm{A}, \mathrm{N}$

Amphiachyris dracunculoides (DC.) Nutt.

prairie broomweed; F or $\mathrm{H}, \mathrm{A}, \mathrm{N}$
Artemisia ludoviciana Nutt.

white sagebrush; F or H, P, N

Ageratina altissima (L.) King \& H.E.

Robins.

white snakeroot; F or $\mathrm{H}, \mathrm{P}, \mathrm{N}$

Antennaria parlinii Fern.

Parlin's pussytoes; F or H, P, N

Bidens bipinnata $\mathrm{L}$.

Spanish needles; F OR H, A, N

Brickellia eupatorioides (L.) Shinners

false boneset; F or H, P, N

Chrysopsis pilosa Nutt.

soft goldenaster; F or $\mathrm{H}, \mathrm{A}, \mathrm{N}$

Cirsium altissimum (L.) Hill

tall thistle; $\mathrm{F}$ or $\mathrm{H}, \mathrm{P}, \mathrm{N}$

Conyza canadensis (L.) Cronq.

Canadian horseweed; F or H, A, N

Coreposis grandiflora Hogg ex Sweet

largeflower tickseed; F or H, P, N

Coreopsis tinctoria Nutt.

golden tickseed; F or $\mathrm{H}, \mathrm{A}, \mathrm{N}$

Dracopis amplexicaulis (Vahl) Cass.

clasping coneflower; F or H, A, N

Eclipta prostrata (L.) L.

false daisy; $\mathrm{F}$ or $\mathrm{H}, \mathrm{A}, \mathrm{N}$

Elephantopus carolinianus

Raeusch.Carolina elephantsfoot;

F or H, P, N

Erigeron tenuis Torr. \& Gray slenderleaf fleabane; F or H, P, N

Eupatorium altissimum L.

tall thoroughwort; F or $\mathrm{H}, \mathrm{P}, \mathrm{N}$

Eupatorium serotinum Michx.

lateflowering thoroughwort;

$\mathrm{F}$ or $\mathrm{H}, \mathrm{P}, \mathrm{N}$

Gaillardia pulchella Foug.

firewheel; F or H, A, N

Gamochaeta purpurea (L.) Cabrera

spoonleaf purple everlasting;

F or $\mathrm{H}, \mathrm{P}, \mathrm{N}$

Grindelia papposa Nesom \& Suh

Spanish gold; F or H, A, N

Helenium amarum (Raf.) H. Rock

Hoagland, B.W. and Buthod, A.K. 
Oklahoma Native Plant Record

Volume 3, Number 1, December 2003

Yellowdicks; F or H, A, N

Helianthus annuus L. common sunflower; F or H, A, N

Helianthus hirsutus Raf. hairy sunflower; F or $\mathrm{H}, \mathrm{P}, \mathrm{N}$

Helianthus maximiliani Schrad. Maximilian sunflower; F or H, P, N

Helianthus petiolaris Nutt. prairie sunflower; F or $\mathrm{H}, \mathrm{A}, \mathrm{N}$

Helianthus tuberosus L. Jerusalem artichoke; F or H, P, N

Heterotheca subaxillaris (Lam.) Britt. \& Rusby camphorweed; F or $\mathrm{H}, \mathrm{A}, \mathrm{N}$

Hieracium longipilum Torr. hairy hawkweed; F or H, P, N

Krigia caespitosa (Raf.) Chambers weedy dwarfdandelion; F or $\mathrm{H}, \mathrm{A}, \mathrm{N}$

Lactuca canadensis L.

Canada lettuce; F or H, A, N

Lactuca floridana (L.) Gaertn. woodland lettuce; F or $\mathrm{H}, \mathrm{A}, \mathrm{N}$

Liatris aspera Michx. tall blazingstar; F or $\mathrm{H}, \mathrm{P}, \mathrm{N}$

Liatris punctata Hook. dotted blazingstar; F or H, P, N

Oligoneuron rigidum (L.) Small stiff goldenrod; F or $\mathrm{H}, \mathrm{P}, \mathrm{N}$

Parthenium hysterophorus L. Santia Maria feverfew; F or H, A, I

Plucea odorata (L.) Cass. sweetscent; F or H, A, N

Pseudognaphalium obtusifolium (L.) Hilliard \& Burtt rabbittobacco; F or $\mathrm{H}, \mathrm{A}, \mathrm{N}$

Pyrrhopappus carolinianus (Walt.) DC. Carolina desert-chicory; F or H, A, $\mathrm{N}$

Ratibida columnifera (Nutt.)

Woot. \& Standl.

upright prairie coneflower;

F or H, P, N

Rudbeckia hirta L. blackeyed Susan; F or H, P, N

Solidago gigantea Ait. giant goldenrod; $\mathrm{F}$ or $\mathrm{H}, \mathrm{P}, \mathrm{N}$

Sonchus asper (L.) Hill spiny sowthistle; F or $\mathrm{H}, \mathrm{A}, \mathrm{I}$

Symphyotrichum drummondii (Lindl.)

Nesom

Drummond's aster; F or H, P, N

Symphyotrichum ericoides (L.) Nesom white heath aster; F or H, P, N

Symphyotrichum oolentangiense (Riddell) Nesom

skyblue aster; F or $\mathrm{H}, \mathrm{P}, \mathrm{N}$

Symphyotrichum patens (Ait.) Nesom late purple aster; F or $\mathrm{H}, \mathrm{P}, \mathrm{N}$

Symphyotrichum subulatum (Michx.)

Nesom

eastern annual saltmarsh aster; F or $\mathrm{H}, \mathrm{A}, \mathrm{N}$

Taraxacum officinale G.H. Weber ex Wiggers common dandelion; $\mathrm{F}$ or $\mathrm{H}, \mathrm{P}, \mathrm{I}$

Thelesperma ambiguum Gray Colorado greenthread; F or H, P, N

Thelesperma filifolium (Hook.) Gray stiff greenthread; F or $\mathrm{H}, \mathrm{P}, \mathrm{N}$

Tragopogon dubius Scop. yellow salsify; F or H, A, I

Verbesina virginica $\mathrm{L}$. white crownbeard; F or $\mathrm{H}, \mathrm{P}, \mathrm{N}$

Vernonia baldwinii Torr. Baldwin's ironweed; F or H, P, N Xanthium strumarium L. rough cocklebur; $\mathrm{F}$ or $\mathrm{H}, \mathrm{A}, \mathrm{N}$

\section{Bignoniaceae}

Campsis radicans (L.) Seem. ex Bureau trumpet creeper; V, P, N

\section{Boraginaceae}

Buglossoides arvensis (L.) I.M. Johnston corn gromwell; F or $\mathrm{H}, \mathrm{A}, \mathrm{I}$

Heliotropium curassavicum L. 
salt heliotrope; F or $\mathrm{H}, \mathrm{A}, \mathrm{N}$

Heliotropium indicum L.

Indian heliotrope; F or H, A, I

\section{Brassicaceae}

Arabis canadensis L. sicklepod; F or H, B, N

Capsella bursa-pastoris (L.) Medik. shepherd's purse; F or H, A, I

Cardamine parviflora $\mathrm{L}$. sand bittercress; F or H, A, N

Draba brachycarpa Nutt. ex Torr. \& Gray shortpod draba; F or $\mathrm{H}, \mathrm{A}, \mathrm{N}$

Draba reptans (Lam.) Fern. Carolina draba; F or $\mathrm{H}, \mathrm{A}, \mathrm{N}$

Erysimum repandum $\mathrm{L}$. spreading wallflower; F or $\mathrm{H}, \mathrm{A}, \mathrm{I}$

Lepidium densiflorum Schrad.

common pepperweed; F or $\mathrm{H}, \mathrm{A}, \mathrm{N}$

Lepidium virginicum L.

Virginia pepperwood; F or $\mathrm{H}, \mathrm{A}, \mathrm{N}$

Rorippa palustris (L.)

bog yellowcress; F or $\mathrm{H}, \mathrm{A}, \mathrm{N}$

Selenia aurea Nutt.

golden selenia; F or $\mathrm{H}, \mathrm{A}, \mathrm{N}$

Cactaceae

Opuntia macrorhiza Engelm. twistspine pricklypear; S, P, N

\section{Campanulaceae}

Triodanis perfoliata (L.) Nieuwl. clasping Venus' looking-glass;

F or $\mathrm{H}, \mathrm{A}, \mathrm{N}$

\section{Caprifoliaceae}

Lonicera japonica Thunb. Japanese honeysuckle; V, P, I

Symphoricarpos orbiculatus Moench coralberry; S, P, N

Viburnum rufidulum Raf. rusty blackhaw; T, S, P, N

\section{Caryophyllaceae}

Arenaria serpyllifolia L. thymeleaf sandwort; ; F or H, A, I

Cerastium glomeratum Thuill. sticky chickweed; F or H, A, I

Dianthus armeria L.

Deptford pink; F or H, A, I

Holosteum umbellatum L.

jagged chickweed; F or H, A, I

Sagina decumbens (Ell.) Torr. \& Gray

trailing pearlwort; F or $\mathrm{H}, \mathrm{A}, \mathrm{N}$

Scleranthus annuus L.

German knotgrass; F or H, A, I

Silene antirrhina $\mathrm{L}$.

sleepy silene; F or $\mathrm{H}, \mathrm{A}, \mathrm{N}$

Stellaria media (L.) Vill. common chickweed; F or H, A, I

\section{Celastraceae}

Celastrus scandens L.

American bittersweet; V, P, N

\section{Chenopodiaceae}

Chenopodium ambrosioides L.

Mexican tea; F or H, A, I

Chenopodium leptophyllum (Moq.) Nutt. ex

S. Wats. narrowleaf goosefoot;

F or $\mathrm{H}, \mathrm{A}, \mathrm{N}$

\section{Cistaceae}

Lechea mucronata Raf.

hairy pinweed; F or $\mathrm{H}, \mathrm{P}, \mathrm{N}$

Lechea tenuifolia Michx. narrowleaf pinweed; $\mathrm{F}$ or $\mathrm{H}, \mathrm{P}, \mathrm{N}$

\section{Clusiaceae}

Hypericum punctatum Lam. spotted St. Johnswort; F or H, P, N

\section{Convolvulaceae}

Convolvulus arvensis L.

field bindweed; F or H, P, I

Ipomoea hederacea Jacq. ivyleaf morning-glory; F or $\mathrm{H}, \mathrm{A}, \mathrm{I}$

Ipomoea lacunosa L. whitestar; F or H, A, N

\section{Cornaceae}

Cornus drummondii C.A. Mey. roughleaf dogwood; T, S, P, N

\section{Cucurbitaceae}

Melothria pendula L.

Hoagland, B.W. and Buthod, A.K. 
Oklahoma Native Plant Record

Volume 3, Number 1, December 2003

Tragia betonicifolia Nutt.

Guadeloupe cucumber; F or H, P, N

Sicyos angulatus L.

oneseed burr cucumber;

F or $\mathrm{H}, \mathrm{A}, \mathrm{N}$

\section{Ebenaceae}

Diospyros virginiana L. common persimmon; T, P, N

\section{Euphorbiaceae}

Acalypha monococca (Engelm. ex Gray) L. Mill. \& Gandhi

slender threeseed mercury;

F or $\mathrm{H}, \mathrm{A}, \mathrm{N}$

Acalypha ostryifolia Riddell

pineland threeseed mercury;

F or $\mathrm{H}, \mathrm{A}, \mathrm{N}$

Acalypha rhomboidea Raf.

Virginia threeseed mercury;

F or $\mathrm{H}, \mathrm{A}, \mathrm{N}$

Chamaesyce maculata (L.) Small spotted sandmat; F or $\mathrm{H}, \mathrm{A}, \mathrm{N}$

Chamaesyce nutans (Lag.) Small eyebane; $\mathrm{F}$ or $\mathrm{H}, \mathrm{A}, \mathrm{N}$

Chamaesyce serpens (Kunth) Small matted sandmat; F or $\mathrm{H}, \mathrm{A}, \mathrm{N}$

Cnidoscolus texanus (Muell.-Arg.) Small Texas bullnettle; F or H, P, N

Croton glandulosus L. vente conmigo; F or $\mathrm{H}, \mathrm{A}, \mathrm{N}$

Croton monanthogynus Michx. prairie tea; $\mathrm{F}$ or $\mathrm{H}, \mathrm{A}, \mathrm{N}$

Croton texensis (Klotzsch) Muell.-Arg. Texas croton; F or $\mathrm{H}, \mathrm{A}, \mathrm{N}$

Euphorbia cyathophora Murr. fire on the mountain; $\mathrm{F}$ or $\mathrm{H}, \mathrm{A}, \mathrm{N}$

Euphorbia dentata Michx. toothed spurge; F or H, A, N,

Euphorbia hexagona Nutt. ex Spreng. sixangle spurge; F or $\mathrm{H}, \mathrm{A}, \mathrm{N}$

Euphorbia marginata Pursh snow on the mountain; F or H, A, N

Stillingia sylvatica Garden ex L. queen's-delight; F or H, P, N

betonyleaf noseburn; F or $\mathrm{H}, \mathrm{P}, \mathrm{N}$

Fabaceae

Albizia julibrissin Durazz. silktree; T, S, P, I

Amorpha canescens Pursh leadplant; SS, S, P, N

Amorpha fruticosa $\mathrm{L}$. desert false indigo; S, P, N

Chamaecrista fasciculata (Michx.) Greene sleeping plant; F or $\mathrm{H}, \mathrm{A}, \mathrm{N}$

Dalea candida Michx. ex Willd. white prairie clover; $\mathrm{F}$ or $\mathrm{H}, \mathrm{P}, \mathrm{N}$

Desmanthus illinoensis (Michx.) MacM. ex B.L. Robins. \& Fern. prairie bundleflower; F or $\mathrm{H}, \mathrm{P}, \mathrm{N}$ Desmodium ciliare (Muhl. ex Willd.) DC. hairy smallleaf ticktrefoil; F or $\mathrm{H}, \mathrm{P}, \mathrm{N}$

Desmodium laevigatum (Nutt.) DC. smooth ticktrefoil; F or $\mathrm{H}, \mathrm{P}, \mathrm{N}$

Desmodium nudiflorum (L.) DC. nakedflower ticktrefoil; F or $\mathrm{H}, \mathrm{P}, \mathrm{N}$

Desmodium obtusum

(Muhl. ex Willd.) DC. stiff ticktrefoil; F or H, P, N

Desmodium paniculatum (L.) DC. panicledleaf ticktrefoil; F or $\mathrm{H}, \mathrm{P}, \mathrm{N}$

Desmodium sessilifolium (Torr.)

Torr. \& Gray sessileleaf ticktrefoil; F or H, P, N

Galactia volubilis (L.) Britt. downy milkpea; F or $\mathrm{H}, \mathrm{P}, \mathrm{N}$

Gleditsia triacanthos L. honeylocust; T, S, P, N

Gymnocladus dioicus (L.) K. Koch Kentucky coffeetree; T, P, N

Lathyrus hirsutus L.

Caley pea; F or H, A, I

Lathyrus pusilllus Ell. tiny pea; F or $\mathrm{H}, \mathrm{A}, \mathrm{N}$

Lespedeza capitata Michx. roundhead lespedeza; F or H, P, N 
Lespedeza stuevei Nutt. tall lespedeza; F or $\mathrm{H}, \mathrm{P}, \mathrm{N}$

Medicago lupulina L. black medick; F or H, P, I

Medicago sativa L. alfalfa; F or H, P, I

Melilotus officinalis (L.) Lam. yellow sweetclover; F or H, A, I

Psoralidium tenuiflorum (Pursh) Rydb. slimflower scurfpea; F or H, P, N

Robinia pseudoacacia L. black locust; T, P, N

Strophostyles helvula (L.) Ell. trailing fuzzybean; F or $\mathrm{H}, \mathrm{A}, \mathrm{N}$

Trifolium arvense L. rabbitfoot clover; F or H, A, I

Trifolium campestre Schreb. field clover; F or $\mathrm{H}, \mathrm{A}, \mathrm{I}$

Trifolium vesiculosum Savi arrowleaf clover; F or $\mathrm{H}, \mathrm{A}, \mathrm{I}$

Vicia sativa L. golden vetch; V, F or H, A, I

Vicia villosa Roth winter vetch: V, F or H, A, I

\section{Fagaceae}

Quercus falcata Michx. southern red oak; T, P, N

Quercus macrocarpa Michx. bur oak; T, S, P, N

Quercus marilandica Muench. blackjack oak; T, S, P, N

Quercus muehlenbergii Engelm. chinkapin oak; T, P, N

Quercus shumardii Buckl. Shumard's oak; T, S, P, N

Quercus stellata Wangenh. post oak; T, P, N

Quercus velutina Lam. black oak; T, P, N

\section{Fumariaceae}

Corydalis micrantha (Engelm. Ex Gray) Gray smallflower fumewort; F or $\mathrm{H}, \mathrm{A}, \mathrm{N}$

\section{Gentianaceae}

Sabatia campestris Nutt. Texas star; F or H, A, N

Geraniaceae

Geranium carolinianum L. Carolina geranium; F or $\mathrm{H}, \mathrm{A}, \mathrm{B}, \mathrm{N}$

Geranium molle L. dovefoot geranium; F or H, A, I

\section{Hydrophyllaceae}

Phacelia strictiflora (Engelm. \& Gray) Gray prairie phacelia; F or $\mathrm{H}, \mathrm{A}, \mathrm{N}$

\section{Juglandaceae}

Carya alba (L.) Nutt. ex Ell. mockerknut hickory; T, $\mathrm{P}, \mathrm{N}$

Carya illinoinensis (Wangenh.) K. Koch pecan; T, P, N

Carya texana Buckl. black hickory; T, P, N

Juglans nigra L. black walnut; T, P, N

\section{Lamiaceae}

Hedeoma hispida Pursh rough false pennyroyal; $\mathrm{F}$ or $\mathrm{H}, \mathrm{A}, \mathrm{N}$

Lamium amplexicaule L. henbit deadnettle; F or H, A, I

Lamium purpureum L. purple deadnettle; F or H, A, I

Monarda fistulosa L. wild bergamot; F or $\mathrm{H}, \mathrm{P}, \mathrm{N}$

Monarda punctata L. spotted beebalm; F or H, A, N

Salvia azurea Michx. ex Lam. azure blue sage; F or $\mathrm{H}, \mathrm{P}, \mathrm{N}$

Teucrium canadense L. Canada germander; $\mathrm{F}$ or $\mathrm{H}, \mathrm{P}, \mathrm{N}$

\section{Lythraceae}

Ammannia coccinea Rottb. valley redstem; F or $\mathrm{H}, \mathrm{A}, \mathrm{N}$

Lythrum alatum Pursh winged lythrum; F or H, P, N

Malvaceae Abutilon theophrasti Medik.

Hoagland, B.W. and Buthod, A.K. 
velvetleaf; F or H, A, I

Callirhoe alcaeoides (Michx.) Gray light poppymallow; F or $\mathrm{H}, \mathrm{P}, \mathrm{N}$

Callirhoe involucrata (Torr. \& Gray) Gray purple poppymallow; F or $\mathrm{H}, \mathrm{P}, \mathrm{N}$

Sida spinosa $\mathrm{L}$. prickly fanpetals; F or $\mathrm{H}, \mathrm{A}, \mathrm{N}$

\section{Menipsermaceae}

Cocculus carolinus (L.) DC.

Carolina coralbead; V, S, P, N

\section{Molluginaceae}

Mollugo verticillata $\mathrm{L}$. green carpetweed; $\mathrm{F}$ or $\mathrm{H}, \mathrm{A}, \mathrm{N}$

Moraceae

Maclura pomifera (Raf.) Schneid. osage orange; T, S, P, N

Morus alba L. white mulberry; T, S, P, I

Nyctaginaceae

Mirabilis nyctaginea (Michx.) MacM. heartleaf four o'clock; F or H, P, N

\section{Oleaceae}

Fraxinus americana $\mathrm{L}$. white ash; T, P, N

\section{Onagraceae}

Gaura longiflora Spach longflower beeblossom; F or H, A, N

Gaura mollis James velvetweed; F or H, A, N

Ludwigia peploides (Kunth) Raven floating primrosewillow; $\mathrm{F}$ or $\mathrm{H}, \mathrm{P}$, $\mathrm{N}$

Oenothera jamesii Torr. \& Gray trumpet evening primrose; F or $\mathrm{H}, \mathrm{P}, \mathrm{N}$

Oenothera rhombipetala Nutt. ex Torr. \& Gray fourpoint evening primrose; F or $\mathrm{H}, \mathrm{P}, \mathrm{N}$

Oxalidaceae

Oxalis stricta L. common yellow oxalis; F or H, P, N

Oxalis violacea $\mathrm{L}$. violet wood sorrel; F or $\mathrm{H}, \mathrm{P}, \mathrm{N}$

\section{Passifloraceae}

Passiflora incarnata $\mathrm{L}$. purple passionflower; F or $\mathrm{H}, \mathrm{P}, \mathrm{N}$ Passiflora lutea $\mathrm{L}$. yellow passionflower; F or $\mathrm{H}, \mathrm{P}, \mathrm{N}$

\section{Phytolaccaceae}

Phytolacca americana $\mathrm{L}$.

American pokeweed; F or $\mathrm{H}, \mathrm{P}, \mathrm{N}$

\section{Plantaginaceae}

Plantago aristata Michx.

largebracted plantain; F or H, A, N

Plantago patagonica Jacq. woolly plantain; F or $\mathrm{H}, \mathrm{A}, \mathrm{N}$

Plantago rhodosperma Dene. redseed plantain; F or $\mathrm{H}, \mathrm{A}, \mathrm{N}$

Plantago rugelii Dene. blackseed plantain; F or H, P, N

\section{Platanaceae}

Platanus occidentalis $\mathrm{L}$.

American sycamore; T, P, N

\section{Polemoniaceae}

Phlox pilosa $\mathrm{L}$. downy phlox; F or H, P, N

\section{Polygalaceae}

Polygala incarnata L.

\section{Polygonaceae}

procession flower; $\mathrm{F}$ or $\mathrm{H}, \mathrm{A}, \mathrm{N}$

Polygonum amphibium L.

water knotweed; F or H, P, N

Polygonum aviculare $\mathrm{L}$.

prostrate knotweed; F or H, A, I

Polygonum lapathifolium L.

curlytop knotweed; F or H, A, N

Polygonum persicaria L.

spotted ladysthumb; F or H, A, N

Polygonum punctatum Ell.

dotted smartweed; F or H, A, N

Polygonum ramosissimum Michx. 
bushy knotweed; F or H, A, N

Polygonum scandens L.

climbing false buckwheat;

F or $\mathrm{H}, \mathrm{P}, \mathrm{N}$

Polygonum virginianum $\mathrm{L}$.

jumpseed; F or $\mathrm{H}, \mathrm{P}, \mathrm{N}$

Rumex crispus L.

curly dock; F or H, P, I

Rumex hastatulus Baldw.

heartwing sorrel; F or H, P, N

Rumex pulcher L.

fiddle dock; F or H, P, I

\section{Portulacaceae}

Claytonia virginica $\mathrm{L}$.

Virginia springbeauty; F or H, P, N

Portulaca oleracea L.

little hogweed; F or H, A, N

\section{Primulaceae}

Androsace occidentalis Pursh

western rockjasmine; $\mathrm{F}$ or $\mathrm{H}, \mathrm{A}, \mathrm{N}$

\section{Ranunculuaceae}

Delphinium carolinianum Walt.

Carolina larkspur; F or H, P, N

Myosurus minimus L.

tiny mousetail; F or $\mathrm{H}, \mathrm{A}, \mathrm{N}$

Ranunculus abortivus L.

littleaf buttercup; F or H, P, N

Ranunculus sceleratus L.

cursed buttercup; F or $\mathrm{H}, \mathrm{A}, \mathrm{N}$

Rosaceae

Geum canadense Jacq. white avens; F or H, P, N

Prunus angustifolia Marsh.

Chickasaw plum; T, S, P, N

Prunus mexicana S. Wats.

Mexican plum; T, S, P, N

Prunus serotina Ehrh.

black cherry; T, S, P, N

Rubus trivialis Michx.

southern dewberry; S, V, P, N

\section{Rubiaceae}

Cephalanthus occidentalis L. common buttonbush; T, S, P, N

Diodia teres Walt. poorjoe; F or $\mathrm{H}, \mathrm{A}, \mathrm{P}, \mathrm{N}$

Diodia virginiana $\mathrm{L}$.

Virginia buttonweed; F or $\mathrm{H}, \mathrm{A}, \mathrm{N}$

Galium aparine L. stickywilly; F or $\mathrm{H}, \mathrm{A}, \mathrm{N}$

Galium pilosum Ait. hairy bedstraw; F or $\mathrm{H}, \mathrm{P}, \mathrm{N}$

Galium virgatum Nutt. southwestern bedstraw; F or H, A, N

Houstonia pusilla Schoepf tiny bluet; F or H, A, N

Saliaceae

Populus deltoides Bartr. ex Marsh. eastern cottonwood; T, P, N

Salix nigra Marsh. black willow; T, P, N

\section{Sapindaceae}

Cardiospermum halicacabum $\mathrm{L}$. love in a puff; F or $\mathrm{H}, \mathrm{A}, \mathrm{N}$

Sapindus saponaria L. var. drummondii (Hook. \& Arn.) L. Benson western soapberry; T, S, P, N

\section{Sapotaceae}

Sideroxylon lanuginosum Michx. gumbully; T, S, P, N

\section{Scrophulariaceae}

Castilleja indivisa Engelm. entireleaf Indian paintbrush; F or $\mathrm{H}, \mathrm{A}, \mathrm{N}$

Leucospora multifida (Michx.) Nutt. narrowleaf paleseed; F or $\mathrm{H}, \mathrm{A}, \mathrm{N}$

Lindernia dubia (L.) Pennell yellowseed false pimpernel; F or $\mathrm{H}, \mathrm{A}, \mathrm{N}$

Nuttallanthus texanus (Scheele) D.A. Sutton Texas toadflax; F or H, A, N

Scrophularia marilandica L. carpenter's square; F or H, P, N 
Verbascum thapsus L. common mullein; F or H, B, I

Veronica polita Fries

Solanaceae gray field speedwell; $\mathrm{F}$ or $\mathrm{H}, \mathrm{A}, \mathrm{I}$

Physalis angulata L. cutleaf groundcherry; F or H, A, N

Physalis heterophylla Nees clammy groundcherry; F or $\mathrm{H}, \mathrm{P}, \mathrm{N}$

Solanum carolinense L.

Carolina horsenettle; F or H, P, N

Solanum dimidiatum Raf. western horsenettle; F or H, P, N

Solanum elaegnifolium Cav. silverleaf nightshade; F or $\mathrm{H}, \mathrm{P}, \mathrm{N}$

Solanum ptychanthum Dunal

West Indian nightshade; F or H,A,N

Solanum rostratum Dunal buffalobur nightshade; F or H, A, N

\section{Tamaricaceae}

Tamarix chinensis Lour.

fivestamen tamarisk; T, S, P, I

Ulmaceae

Celtis laevigata Willd. sugarberry; T, S, P, N

Ulmus americana L.

American elm; T, P, N

Ulmus rubra Muhl.

Urticaceae slippery elm; T, P, N

Laportea canadensis (L.) Weddell

Canadian woodnettle; F or H, P, N

Parietaria pensylvanica Muhl. ex Willd.

Pennyslvania pellitory; F or $\mathrm{H}, \mathrm{A}, \mathrm{N}$

Valerianaceae

Valerianella radiata (L.) Dufr.

\section{beaked cornsalad; F or H, A, N}

\section{Verbenaceae}

Glandularia canadensis (L.) Nutt. rose mock vervain; $\mathrm{F}$ or $\mathrm{H}, \mathrm{P}, \mathrm{N}$

Glandularia pumila (Rydb.) Umber pink mock vervain; $\mathrm{F}$ or $\mathrm{H}, \mathrm{A}, \mathrm{N}$

Phyla nodiflora (L.) Greene

turkey tangle fogfruit; F or H, P, N

Verbena stricta Vent. hoary verbena; F or $\mathrm{H}, \mathrm{P}, \mathrm{N}$

Verbena urticifolia L. white vervain; F or $\mathrm{H}, \mathrm{P}, \mathrm{N}$

\section{Violaceae}

Viola affinis Le Conte sand violet; F or $\mathrm{H}, \mathrm{P}, \mathrm{N}$

Viola bicolor Pursh

Vitaceae

field pansy; F or $\mathrm{H}, \mathrm{A}, \mathrm{N}$

Ampelopsis cordata Michx. heartleaf peppervine; $\mathrm{V}, \mathrm{P}, \mathrm{N}$

Cissus trifoliata (L.) L. sorrelvine: V, SS, P, N

Parthenocissus quinquefolia (L.) Planch. Virginia creeper; V, P, N

Vitis cinera (Engelm.) Millard graybark grape; V, P, N

Vitis riparia Michx. riverbank grape; V, P, N

Vitis vulpina L. frost grape; V, P, N

\section{Zygophyllaceae}

Tribulus terrestris L.

puncturevine; F or $\mathrm{H}, \mathrm{A}, \mathrm{I}$ 